\title{
Using Mental Health Podcasts for Public Education
}

\author{
Cheryl D. Wills ${ }^{1}$
}

Received: 30 January 2020 / Accepted: 17 June 2020 / Published online: 9 July 2020

(C) Academic Psychiatry 2020

Advances in medical and technological knowledge have changed psychiatry graduate medical education. Residents are required to learn and apply volumes of complex information and to become proficient in clinical service delivery to meet the Accreditation Council for Graduate Medical Education (ACGME) core competency requirements in psychiatry [1]. The information gleaned from didactic education, supervision, reading, observation, practicing, collaborative communication, and other education modalities facilitates building and refining a skillset that is a prerequisite for independent practice [2]. Unfortunately, the growing body of professional resources, including medical literature, makes it challenging, at best, to produce well-rounded physician/ scholars in an increasingly complex society.

The psychiatry Residency Training Director is challenged with the task of crafting a training experience that treats education requirements equitably. She prioritizes training opportunities and refines the didactics curriculum and other educational modalities to offer residents a quality and balanced time-limited training experience that meets ACGME standards. This is an onerous task given the growing volume of available educational resources, clinical need, and related opportunities. Consequently, some sectors of required psychiatric education that may merit additional attention may be overlooked [1]. One such area involves training psychiatric residents to function as advocates for the profession and their patients. Residents can refine their communication skills by reaching out to and educating the public about mental health - including emotional wellness, the mental health system, psychiatric assessment and treatment, destigmatizing mental illness, and access to care - in greater detail than what is available in medical office brochures and public service announcements.

Cheryl D. Wills

cwforensic@earthlink.net

1 Case Western Reserve University, Cleveland, OH, USA
One way to accomplish this involves empowering psychiatry residents to use critical thinking skills to identify lowcost, high-quality mental health educational materials that may appeal to the public. This requires residents to use a dispassionate, evidence-informed, and systematic approach to examining publicly accessible mental health resources. Though the Internet offers access to various forms of media, an Internet audio podcast program - an episodic series of serial recordings that are accessible by downloading their digital files - was used for the project because the recordings can be listened to on many types of electronic devices (computers, smartphones, tablets, MP3 players, automobile stereos, compact disk players, etc.), accessed in many settings, listened to while multitasking and replayed, or in some cases bookmarked, so that the user will be able to review specific segments if desired. Additionally, each podcast recording does not have to be consumed in one sitting.

Residents were instructed to listen to several episodes of The Mind Deconstructed Podcast: Mental Health and Wellness with Dr. Kaz and George [3]. The Minnesotabased mental health education program is hosted by siblings Kaz Nelson, M.D., a psychiatrist and psychiatry Residency Training Director, and her brother, George, an attorney who works in child welfare. Their mission is to examine topics in psychiatry, including violence, anxiety, posttraumatic stress disorder, and mental health maintenance, in a manner that is user-friendly for non-healthcare professionals.

Residents used set descriptors while explaining their impressions to their colleagues. The categories included accuracy of the information (evidence-informed), ease of comprehension (organized), language (absence of jargon), presentation style (approachable), length of recordings, and value of information for laypersons (Table 1). Although the free podcast was easily accessed and had good sound quality, these criteria also merit consideration (Table 1). Residents also were also instructed to describe their vision for the future of the podcast and to offer constructive feedback. The residents knew that I would be sharing the feedback with the podcast's hosts. The Case Western Reserve University Institutional 
Table 1 Criteria for podcast evaluations

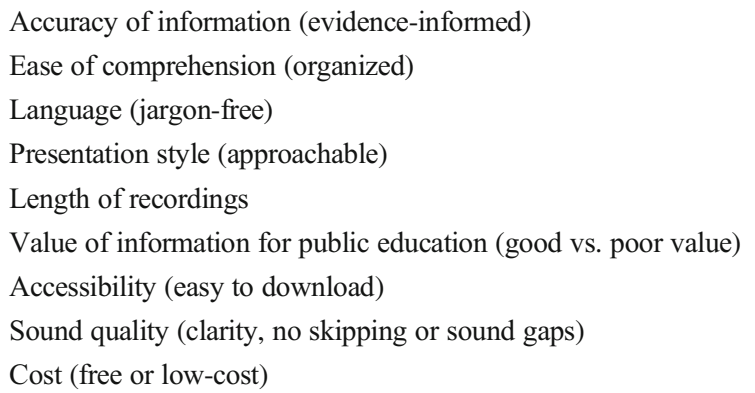

Review Board issued an exemption waiver for the educational exercise and this manuscript.

The residents listened to the recordings while driving, walking, exercising, and relaxing and between work obligations. They were eager to share their observations. All said that the content, which contained substantially more detail than a basic mental health information brochure, was fairly accurate and contained educational content that was suitable for a public audience of adult non-healthcare professionals. The hosts, according to the residents, were welcoming and approachable and had a relaxed communication style. All residents opined that the 16-30-minute recordings were reasonable in length and some said that recordings were pleasant to listen to while the residents were relaxing, exercising, or commuting to or from work.

Several residents said and all concurred that the hosts modeled clear, concise, jargon-free, and non-intimidating ways to convey information about challenging mental health topics to the public, including patients. This motivated some residents to work on refining their communication skills so that they will be more effective when engaging an audience of patients and other non-healthcare professionals. Some residents appreciated the clinical "pearls" that Dr. Kaz offered and said that they planned to educate their patients about the suggested techniques for improving one's relaxation and coping skills. The residents all determined that the recordings would reduce stigma, which can be associated with seeking mental health services, by demystifying mental health, empowering the public to learn more about mental health matters, and legitimizing the expectation that individuals who ask questions to healthcare providers about mental health receive clear, nonjudgmental responses from them.

By all accounts, the critical thinking exercise was valuable. The free podcast recordings were easy for residents to access, listen to, and critique. Residents' feedback included suggestions of possible ways to enhance future segments of The Mind Deconstructed Podcast: Mental Health and Wellness with Dr. Kaz and George, including lightly editing the recordings prior to posting them, using a small lay focus group to provide feedback about the listening experience and asking listeners to suggest future topics for discussion on podcast episodes. Though not required, the suggested modifications could contribute to the podcast being more timely, relevant, and accessible for years to come. Several residents said they look forward to listening to future episodes of the podcast and hoped that the hosts would consider inviting mental health professionals to be guest discussants on the program so that listeners will have access to various perspectives about mental health, mental disorders, and therapeutic interventions.

Psychiatry residents use critical thinking while assimilating knowledge and applying it to care for patients in various ways, including interviewing and diagnosing them, conducting risk assessments, and collaborating with patients to craft individualized treatment plans. In this age of information overload, residents must be capable of appraising mental health education materials and identifying quality, affordable resources for patients and the public. By so doing, the residents can extend the reach of their public mental health advocacy by educating the public about vetted resources that may expand their access to quality mental health educational materials. The podcast appraisal exercise is one way to accomplish this.

The Internet is a major accessible source of low and no-cost mental health information for patients and the public. This includes general information, symptom inventories, sleep logs, and apps to aid with tracking one's mood, learning, and refining relaxation techniques, coping with anxiety, and/ or reducing nicotine consumption. While some of these educational tools are based on evidence-based medicine, others are not. Some resources may actually be harmful due to inaccurate content, data mining, etc. Residents are, with proper guidance, capable of identifying suitable mental health resources for educating laypersons and excluding media that lack accuracy and/or have low-quality content.

Residents who critically examine electronic mental health education materials can collaborate with colleagues, in seminars, in groups, or by email or another form of electronic communication. The group can curate a list of cost-effective resources that are suitable for public mental health education. The residents can cite the material when advocating for the profession - during face-to-face discussions, by mail or via electronic media - with patients, the public, mental health advocacy groups, and non-psychiatrist colleagues.

The critical thinking exercise by which the educational materials are vetted should include a discussion, with residents, about the caveats of recommending any electronically available mental health resources to others for educational purposes. For example, residents should ensure that any discussion about a curated resource is accompanied by a disclaimer: the resident is not endorsing a particular mental health education resource as an authoritative resource. Also, reviewing an educational resource is not a substitute for 
properly undergoing a mental health assessment and/or receiving mental health treatment. Additionally, users should be cautious about providing personal information to a media resource; although there are exceptions, there generally is no guarantee of privacy. Importantly, the content of educational media is subject to change without notice at the discretion of its owner. Lastly, residents who wish to curate their own websites should check with the Training Director about institution policies, training expectations, and the pros and cons of posting information on social media [4].

The podcast evaluation project has been a useful educational and skill building exercise for residents. It is a first step in advocating that residents use clinical knowledge to inform critical thinking to benefit public mental health education. The assignment, which fosters collaboration, professionalism, advocacy, and objectivity, can be used to refine our residents' skillset in training and later, when they are pursuing independent psychiatric practice. The residents' constructive feedback could also, in the future and with proper consent, be used by electronic mental health education content producers to refine the quality and user-friendliness of their products. The activity may even motivate some residents to develop their own mental health podcasts as online teaching tools.

Future plans for the critical thinking activity include reviewing other mental health education podcasts as well as other educational media. This will help the residents identify quality, affordable, and accessible mental health educational programming that may appeal to members of the public who may prefer different media formats and/or have different learning styles. Videos, e-books, questionnaires, checklists, apps, and electronic games can be critiqued by residents, in the future, for their suitability as mental health education tools for public use. Also, interdisciplinary examination of affordable mental health education resources can be used to foster a team approach to public mental health advocacy and education. This concept can be conducive to interdisciplinary collaboration and to residents and participants from other disciplines developing mutual respect for the skillsets of their colleagues.

Using graduate medical education training to refine evaluative skills advances the transformative process by which psychiatry residents become disciplined, dispassionate thinkers capable of providing constructive scholarly, clinical, and practical feedback, to individuals in the academy and the community, and may improve their capacity to become better practitioners after they graduate from residency. Residents who function as public mental health advocates are working to address mental health disparities, as they strive to become independent practitioners.

\section{Compliance with Ethical Standards}

The Case Western Reserve University Institutional Review Board issued an exemption waiver for the educational exercise and this manuscript.

Disclosure The author states that there is no conflict of interest.

\section{References}

1. Accreditation Council for Graduate Medical Education Program Requirements for Graduate Medical Education in Psychiatry. https://www.acgme.org/Portals/0/PFAssets/ProgramRequirements/ 400_psychiatry_2017-07-01.pdf. Accessed 28 Dec 2019.

2. Bromley E, Braslow JT. Teaching critical thinking in psychiatric training: a role for the social sciences. Am J Psychiatry. 2008;165: 1396-401.

3. The mind deconstructed podcast: mental health and wellness with Dr. Kaz and George. http://theminddeconstructed.libsyn.com/. Accessed 28 Dec 2019.

4. DeJong SM, Benjamin S, Anzia JM, et al. Professionalism and the Internet in psychiatry: what to teach and how to teach it. Acad Psychiatry. 2012;36:356-62.

Publisher's Note Springer Nature remains neutral with regard to jurisdictional claims in published maps and institutional affiliations. 